\title{
Long-term spectroscopy of Wolf-Rayet binaries
}

\author{
Jörg Schweickhardt ${ }^{1}$, Werner Schmutz ${ }^{2}$, Andreas Kaufer ${ }^{1}$, Otto Stahl ${ }^{1}$, \\ and Bernhard Wolf ${ }^{1}$ \\ 1 Landessternwarte Königstuhl, D-69117 Heidelberg, Germany \\ 2 Institut für Astronomie, ETH-Zentrum, CH-8092 Zürich, Switzerland
}

\begin{abstract}
We are investigating two long-period $\mathrm{WR}+\mathrm{O}$ binaries, $\gamma^{2}$ Velorum (WC8+07.5, $P=78.5 \mathrm{~d}$ ) and WR $22(\mathrm{WN} 7+\mathrm{abs}+\mathrm{O}, P=80.3 \mathrm{~d})$. We derive new orbital elements for both binaries and we give preliminary results of our analysis of the expansion velocity and ionization structure of $\gamma^{2}$ Vel.
\end{abstract}

\section{Orbital elements and mass determination}

Our analyses are based on high-resolution spectra $(R=20000)$ in the optical range (3506-8700 $\AA$ ) which were collected with the fiber-fed echelle spectrograph HEROS at the ESO 50-cm telescope in 1995 and 1996. Our spectroscopic time series cover more than one full orbital period with a time resolution of 1 day, i.e., steps of 0.013 in phase. From these large observational data sets we derive new orbital solutions and masses for both binaries. In Table 1 we summarize the physical parameters of $\gamma^{2}$ Vel and WR 22 .

Table 1. Orbital parameters of $\gamma^{2}$ Velorum (Schmutz et al. 1997) and WR 22 (Schweickhardt et al. 1999).

\begin{tabular}{l|rl|rl}
\hline star & \multicolumn{2}{|c|}{$\gamma^{2}$ Velorum } & \multicolumn{2}{c}{ WR 22 } \\
spectral type & \multicolumn{2}{|c}{ WC8 + O 7.5} & \multicolumn{2}{c}{ WN7+abs+O } \\
\hline$P$ [days] & 78.53 & \pm 0.01 & 80.336 & \pm 0.0013 \\
$e$ & 0.326 & \pm 0.01 & 0.598 & \pm 0.004 \\
$\gamma\left[\mathrm{km} \mathrm{s}^{-1}\right]$ & 7 & \pm 23 & 0 & \pm 10 \\
$T_{0}[\mathrm{JD} 2400000.5+]$ & 50.0 & \pm 1.6 & 50126.97 & \pm 0.06 \\
$\omega_{\mathrm{WR}}[\mathrm{deg}]$ & 68 & \pm 4 & 268.2 & \pm 0.6 \\
$K_{\mathrm{WR}}\left[\mathrm{km} \mathrm{s}^{-1}\right]$ & 122 & \pm 2 & 70.62 & \pm 0.32 \\
$K_{\mathrm{O}}\left[\mathrm{km} \mathrm{s}^{-1}\right]$ & 38.4 & \pm 2 & 190 & \pm 10 \\
$i[\mathrm{deg}]$ & 65 & \pm 8 & 90 & \\
$m_{\operatorname{mass~ratio~} q}$ & 0.31 & \pm 0.017 & 2.69 & \pm 0.14 \\
$a_{\mathrm{WR}} \sin i\left[10^{6} \mathrm{~km}\right]$ & 124.5 & \pm 2 & 62.5 & \pm 0.4 \\
$a_{\mathrm{O}} \sin i\left[10^{6} \mathrm{~km}\right]$ & 39.2 & \pm 2 & 168.2 & \pm 8.8 \\
$\left(M_{\mathrm{WR}}+M_{\mathrm{O}}\right) \sin ^{3} i\left[\mathrm{M}_{\odot}\right]$ & 28.4 & \pm 1.6 & 75.9 & \pm 8.8 \\
$M_{\mathrm{WR}} \sin { }^{3} i\left[\mathrm{M}_{\odot}\right]$ & 6.8 & \pm 0.6 & 55.3 & \pm 7.2 \\
$M_{\mathrm{O}} \sin { }^{3} i\left[\mathrm{M}_{\odot}\right]$ & 21.6 & \pm 1.1 & 20.6 & \pm 1.6 \\
\hline
\end{tabular}

\section{Effects of wind occultation and colliding winds}

Wind occultation of the O-type star and colliding winds both produce variable line shapes. In order to isolate these effects we first investigate the wind occu- 


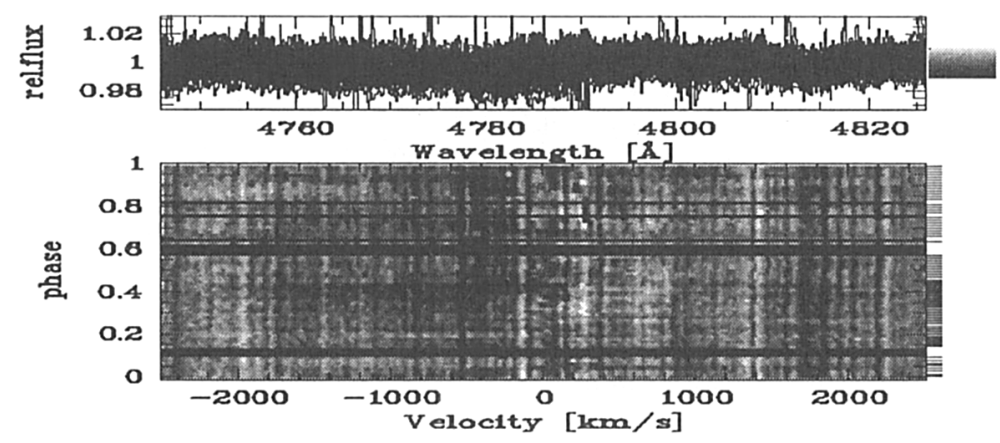

Figure 1. Difference spectra relative to phase 0.67 of $\mathrm{C}$ IV $\lambda 4785.9$ as an example for all weak C IV emission lines.

lation because there is a theory (Auer \& Koenigsberger 1994) that allows us to model theoretically the variability. We calculate the difference of the individual spectra to that at phase 0.67 , when the O-type star is near quadrature, i.e., at maximum impact distance $P$ (we choose here phase 0.0 when the WR is in front of the $\mathrm{O}$ star). In Fig. 1 we show the difference spectra of the C IV $\lambda 4785.9$ emission line. This line is representive for all weak C IV lines, selected because of its isolated position.

Figure 2 shows the difference spectra of the strongest emission line in our spectra: the CIII $\lambda 4650$ complex. The spectra are corrected for the orbital motion of the WR star. The top panel shows an overlay of all difference spectra. The bottom panel shows the same spectra with the $\mathrm{x}$-axis in velocity units and the $y$-axis corresponding to the orbital phase. The intensities are coded in grey scales according to the scale indicated on the right of the top panel.

All weak emission lines of C IV (e.g., Figure 1) show rather flat difference spectra i.e., the remaining signatures are of the order of $1 \%$. No occultation effects or effects of colliding winds are visible. Therefore, the $\mathrm{O}$ star's apparent orbit has to be completely outside the line emitting region of the $\mathrm{CIV}$ lines.

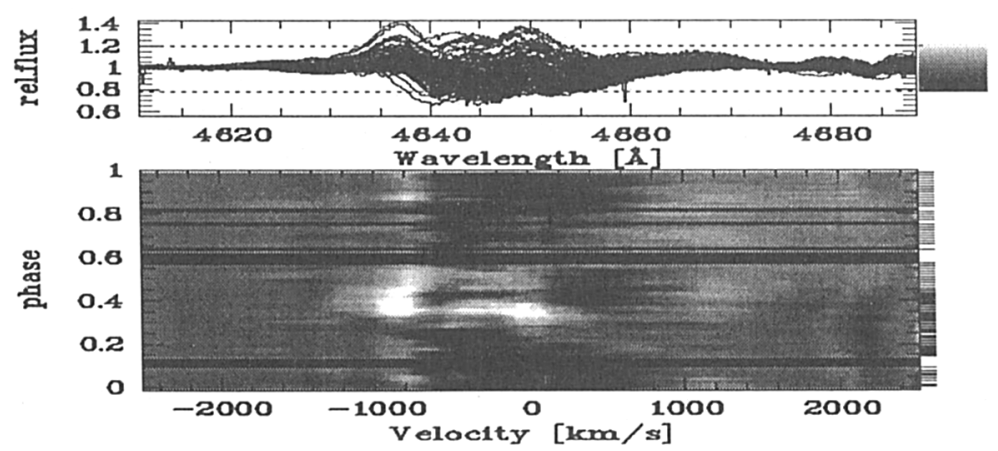

Figure 2. Difference spectra relative to phase 0.67 of $\mathrm{C}$ III $\lambda 4650$, the strongest emission line in our spectra. 


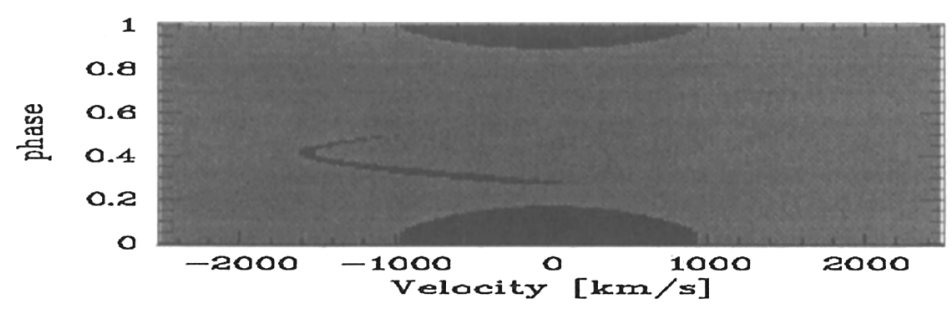

Figure 3. Synthetic difference spectra with wind occultation effects in the $\mathrm{WR}+\mathrm{O}$ binary system $\gamma^{2}$ Velorum.

Based on the orbital elements (Table 1 ), we estimate the size of the emitting region to be inside a radius $r<140 \mathrm{R}_{\odot}$. The line widths of the C IV lines corresponds to $1300-1500 \mathrm{~km} \mathrm{~s}^{-1}$. Therefore, the WR wind must accelerate inside $r<140 \mathrm{R}_{\odot}$ to a velocity of $\sim 1400 \mathrm{~km} \mathrm{~s}^{-1}$.

The strong C III $\lambda 4650$ emission (Fig. 2) shows a different behaviour. We observe symmetric absorption features at phases $0.80-0.20$ and additional emission at phase 0.40 . This coincides with the periastron at phase 0.39 . Therefore, the $\mathrm{O}$ star light interacts with the $\mathrm{C}$ III-line emitting region of the Wolf-Rayet wind.

We simulate this variability with the model of Auer \& Koenigsberger (1994). This simulation takes into account the occulation effects of the $\mathrm{O}$ star shadowing part of the WR wind and the radiation transfer of the $\mathrm{O}$ star radiation through the WR wind. We do not account for any contribution from the colliding wind zone.

Figure 3 shows a simulated dynamical spectrum displayed on the same scale as the observations (Fig. 2). The simulation is made for an inclination angle of $i=57^{\circ}$. We use a velocity law that accelerates linearly with radius from $1400 \mathrm{~km} \mathrm{~s}^{-1}$ at $r=140 \mathrm{R}_{\odot}$ to $1800 \mathrm{~km} \mathrm{~s}^{-1}$ at $r=230 \mathrm{R}_{\odot}$. In our model the $\mathrm{C}_{\text {III }}$ line-emitting region ends at $r=230 \mathrm{R}_{\odot}$.

With this simple model, the observed line-profile variations of Fig. 2 are partially reproduced by our synthetic spectrum (Fig. 3), in particular the absorption features around phase 0.0. At these positions, wind occultation effects are visible. On the other hand, the bright emission around periastron is not predicted by our model. Instead, on the base of our simulation, we expect a narrow absorption feature to show up in the difference spectrum (here the $\mathrm{O}$ star immerses into the WR wind). This absorption is probably blended by the emission which we believe to originate from the interaction zone of the colliding winds.

\section{References}

Auer, L., Koenigsberger, G. 1994, ApJ 436, 859

Schmutz, W., Schweickhardt, J., Stahl, O. et al. 1997, A\&A 328, 219

Schweickhardt, J., Schmutz, W., Stahl, O. et al. 1999, in preparation 